\section{Modificações da junção uretrovesical e uretra proximal após a cirurgia de alça sem tensão em mulheres de 45 a 72 anos}

\section{Urethrovesical junction and proximal urethra changes following tension-free vaginal tape surgery in women aged 45 to 72 years old}

Frederico Teixeira Brandt 1 Misael Wanderley dos Santos Junior 2 Carla Daisy Costa Albuquerque 3 Felipe Rinald Barbosa Lorenzato 4 Laila Almeida Viana 5 Adriana Scavuzzi Carneiro da Cunha 6

1,2,3,5 Unidade de Pesquisa em Incontinência Urinária. Universidade Federal de Pernambuco. Campus Universitário. Cidade Universitária. Recife, PE, Brasil. CEP: 50.670-420. E-mail: fcbrandt@elogica.com.br

4,6 Instituto Materno Infantil Prof. Fernando Figueira, IMIP. Recife, PE, Brasil.

\begin{abstract}
Objectives: to describe the urethrovesical junc tion and proximal urethra changes following Tensionfree Vaginal Tape (TVT) procedure for stress urinary incontinence treatment (SUI).

Methods: this study was performed at the Urinary Incontinence Research Unit of the Universidade Federal de Pernambuco. Twenty two stress urinary incontinence female patients were submitted to TVT. They were evaluated through ultrasound before and following surgery.

Results: ultrasound results at rest, before and following TVT surgery, were: mean displacement of urethrovesical junction vertical length, before and after TVT procedure respectively $16,7 \mathrm{~mm}$ and 10,7 $\mathrm{mm}$; and mean displacement of proximal urethra length, before and following TVT surgery, respectively $13,1 \mathrm{~mm}$ and $6,7 \mathrm{~mm}$.

Conclusions: TVT surgery used for treating women with stress urinary incontinence significantly reduces the vertical mobility of the urethrovesical junction $(p=0,005)$ and proximal urethra $(p=0,02)$.
\end{abstract}

Key words Urinary incontinence, stress, Surgery, Women

\section{Resumo}

Objetivos: descrever as modificações da junção uretrovesical e uretra proximal decorrentes do uso do Tension-Free Vaginal Tape (TVT), empregado no tratamento da incontinência urinária de esforço (IUE).

Métodos: trabalho realizado na Unidade de Pesquisa em Incontinência Urinária da Universidade Federal de Pernambuco. Foram estudadas 22 mulheres com incontinência urinária de esforço foram submetidas à ciru rgia do tipo TVT e avaliadas por ultra-sonografia antes e depois da cirurgia.

Resultados: os deslocamentos verticais médios da distância vertical da junção uretrovesical no pré e pós-operatório foram respectivamente de $16,7 \mathrm{~mm} e$ de 10,7 mm; e os deslocamentos médios da uretra proximal no pré e pós-operatório foram de $13,1 \mathrm{~mm}$ e de $6,7 \mathrm{~mm}(p=0,02)$.

Conclusões: a cirurgia do tipo TVT, usada no tratamento de mulheres com incontinência urinária de esforço, reduz significativamente a mobilidade vertical da junção uretrovesical $(p=0,005)$ e da uretra proximal $(p=0,02)$.

Palavras-chave Incontinência urinária de esforço, Cirurgia, Mulheres 


\section{Introdução}

A incontinência urinária feminina atinge milhões de mulheres e tem alta prevalência no mundo, chegando a $28 \%$ em alguns países da Europa. 1

A relevância desse problema se reflete nos cerca de 15 bilhões de dólares gastos anualmente, em países da Europa e Estados Unidos, no diagnóstico e tratamento desse tipo de incontinência e no impacto na qualidade de vida dessas mulheres, levando a queda da auto-estima, constrangimento e isolamento social. ${ }^{2}$

Apenas 25\% das pacientes com algum grau de incontinência urinária, e cerca de metade das que têm incontinência significativa, procuram consulta médica a respeito do problema. 1,2

$\mathrm{Na}$ atualidade, entre os vários tratamentos preconizados para ao tratamento da incontinência urinária de esforço (IUE), vem se destacando o uso de determinados tipos de alça tecidual, naturais ou sintéticos, envolvendo a uretra.3-6

$\mathrm{Na}$ última década, entre as diversas alças utilizadas para o tratamento da incontinência urinária de esforço, o Tension-free Vaginal Tape" (TVT) tem tido uma boa preferência entre os cirurgiões. ${ }^{3-6}$

Historicamente, existem alguns questionamentos clássicos com relação ao tratamento cirúrgico da IUE. Quais sejam: a) o tratamento foi eficaz? b) por quanto tempo? c) provocou obstrução? d) o que a cirurgia provoca no posicionamento anatômico e funcional da uretra?3

Revendo a literatura sobre o TVT, utilizado no tratamento da IUE, há controvérsia sobre os três primeiros questionamentos. ${ }^{3-6}$ Entretanto, não está evidente a resposta do que o TVT provoca na junção uretrovesical e na uretra proximal.

Esse artigo tem por finalidade mostrar de forma objetiva as modificações da junção uretrovesical e uretra proximal, decorrentes do uso do TVT, empregado no tratamento da IUE.

\section{Métodos}

Foram estudadas 22 pacientes do sexo feminino no período de março de 2002 a dezembro de 2003, na Unidade de Pesquisa de Incontinência Urinária (UPIU) da Universidade Federal de Pernambuco em Recife, Pernambuco, Brasil.

Todas as pacientes incluídas no estudo procuraram espontaneamente a UPIU ou foram encaminhadas para consulta médica especializada com queixas de IUE e concordaram com o protocolo de pesquisa através da assinatura do termo de consentimento livre e esclarecido.

Os critérios de inclusão na investigação foram: a) mulheres entre 40 e 75 anos; b) ter como queixa principal a perda de urina relacionada a esforço como tossir, espirrar ou levantar-se, há pelo menos seis meses; c) ter perda de urina clinicamente demonstrável ao exame físico, ou fazer uso regular de algum tipo de protetor higiênico; e d) concordar em se submeter ao tratamento cirúrgico proposto (TVT).

Para os critérios de exclusão foram considerados: a) antecedente de cirurgia para IUE nos últimos cinco anos; b) história e exame físico sugestivo de bexiga hiperativa ou neurogênica; c) presença de doença neurológica.

As 22 pacientes estudadas estavam entre a faixa etária de 42 a 72 anos, com uma média de 59 anos.

Os critérios utilizados para diagnóstico de hipermobilidade da junção uretrovesical (JUV) foram os sugeridos por Brandt et al.,7 que usaram o deslocamento vertical da JUV maior que $9,0 \mathrm{~mm}$ como parâmetro de maior precisão, conforme Figura 1 .

O procedimento cirúrgico foi realizado, segundo a técnica originariamente descrita por Ulmsten et al. $5 \mathrm{e}$ modificada como descrito a seguir: com a paciente em posição de litotomia, e sob raquianestesia realizou-se uma incisão vaginal mediana de aproximadamente $1,5 \mathrm{~cm}$ iniciada a cerca de $1,0 \mathrm{~cm}$ do meato uretral em direção proximal, porém sem atingir o colo vesical. A seguir, foi realizada dissecção mínima com tesoura, lateralmente, de ambos os lados da uretra, criando-se um túnel de $1,0 \mathrm{~cm}$, suficiente para introduzir a extremidade da agulha do TVT.

Introduziu-se, por via uretral, um cistoscópio com camisa $17 \mathrm{~F}$, esvaziou-se a bexiga e, na seqüência utilizando-se o próprio cistoscópio deslocou-se a uretra e colo vesical para a posição contra lateral do trajeto da passagem da agulha do TVT. O sling (TVT), que possui duas agulhas de forma arqueada de $5 \mathrm{~mm}$ de diâmetro em cada extremidade, foi então preparado introduzindo-se uma dessas agulhas na manopla metálica e a seguir guiado no trajeto dos túneis previamente dissecados e, avançado, inicialmente em direção horizontal até sentir-se o periósteo inferior do osso púbis. Em seguida através de movimento de báscula por trás do púbis avançou-se superior e lateralmente em direção ao ombro homolateral da paciente até a saída da ponta da agulha no hipogástrio (Figura 2). 
Ultra-sonografia transvulvar da junção uretrovesical (JUV) no repouso e ao esforço.
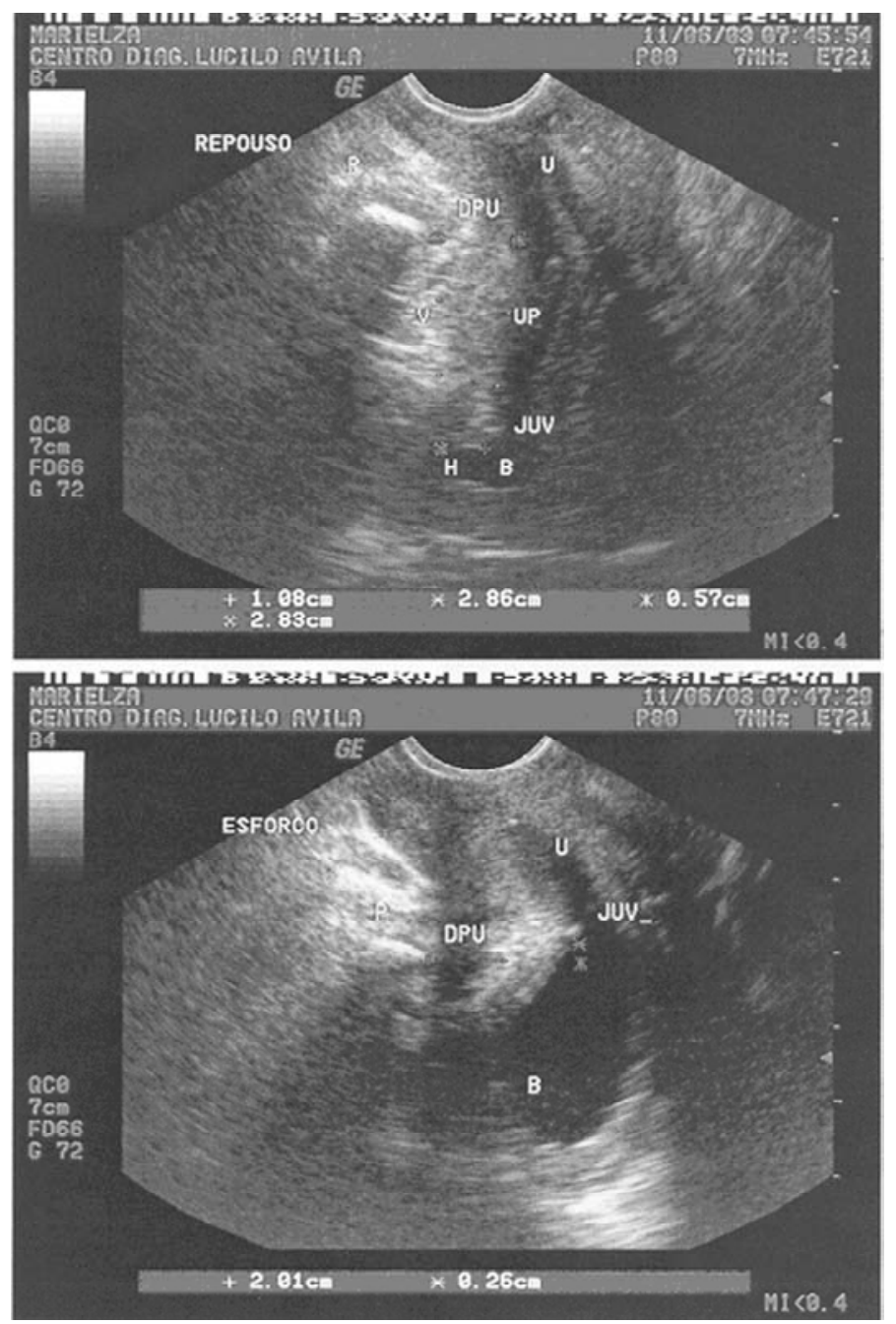

Antes da extrusão completa da agulha do TVT, realizou-se cistoscopia para verificar uma possível perfuração vesical.

As mesmas manobras foram repetidas do outro lado, obtendo-se assim a alça em forma de "U" do sling pubovaginal no terço médio da uretra. Procedeu-se ao ajuste da tensão do TVT e seu posicionamento suburetral antes da remoção dos envelopes plásticos como descrito na técnica original. 5 A profilaxia antimicrobiana foi realizada com cefazolina (1 grama) endovenosomente uma hora antes da cirurgia e mantida a intervalo de 6 horas por 24 horas. Foi utilizado cateter de demora com sistema coletor fechado por 24 horas exceto em caso de perfuração vesical, quando foi mantido por 48 horas.

Os dados da amostra foram analisados no programa Epi Info 6.0, utilizando-se o teste "t" de Student para amostra pareada.

Os resultados das variáveis contínuas foram expressos por suas médias e desvio padrões.

O intervalo de confiança adotado foi de $95 \%$ (IC95\%) e o nível de significância foi considerado quando o valor de $p$ foi menor que $0,05(p<0,05)$.

A pesquisa teve o apoio do Comitê de Ética da Instituição. 
Detalhe da passagem do tension-free vaginal tape (TVT) pelo espaço retropúbico.

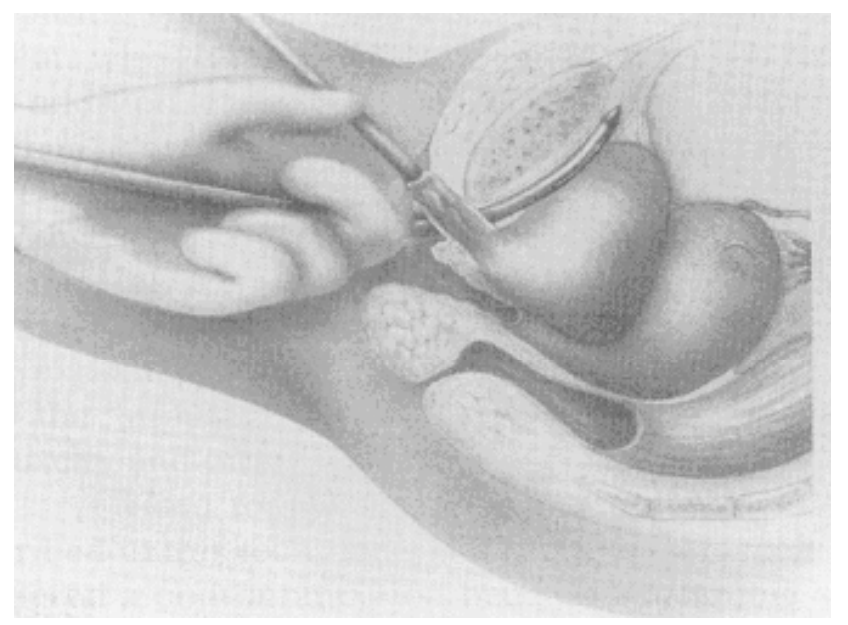

\section{Resultados}

O acompanhamento médio das pacientes foi de 8 meses, variando de 2 a 18 meses. A cirurga de TVT teve uma taxa de sucesso de $91 \%$ em curto prazo (quatro semanas) e de $95,5 \%$ até ao final do período de acompanhamento descrito.

As medidas obtidas pela ultra-sonografia (valores expressos em milímetros), estão apresentadas nas Tabelas 1 a 4 . A média do deslocamento vertical da JUV foi significantemente maior no pré-operatório
$(16,7 \mathrm{~mm})$ de que após a cirurgia $(10,7 \mathrm{~mm})$ $(p=0,005)$. O deslocamento da uretra proximal também foi significantemente alterado após a cirurgia, com uma média de $13,1 \mathrm{~mm}$ no pré-operatório e de 9,2 $\mathrm{mm}$ no pós-operatório $(p=0,02)$. Os outros dois parâmetros estudados, a distância horizontal da junção uretrovesical (DHJUV) e distância pubouretral (DPU), não apresentaram medidas ultrasonográficas de deslocamento estatisticamente significantes após a cirurgia, quando comparados ao pré-operatório.

Tabela 1.

Estatísticos relativas à distância vertical da junção uretrovesical (DVJUV) de 22 mulheres submetidas à cirurgia de tension-free vaginal tape (TVT) para correção da incontinência urinária de esforço.

\begin{tabular}{|c|c|c|c|c|c|c|}
\hline \multirow{2}{*}{ DVJUV } & \multicolumn{2}{|c|}{ Repouso } & \multicolumn{2}{|c|}{ Esforço } & \multicolumn{2}{|c|}{ Deslocamento } \\
\hline & Pré-operatório & Pós-operatório & Pré-operatório & Pós-operatório & Pré-operatório & Pós-operatório \\
\hline Média (mm) & 16,0 & 16,6 & 2,1 & 8,4 & 16,7 & 10,7 \\
\hline Desvio-padrão & 4,6 & 3,2 & 15,3 & 9,6 & 7,7 & 7,3 \\
\hline Significância & \multicolumn{2}{|c|}{$p=0,26$} & \multicolumn{2}{|c|}{$p=0,28$} & \multicolumn{2}{|c|}{$p=0,005$} \\
\hline
\end{tabular}


Estatísticos relativas à distância horizontal da junção uretrovesical (DHJUV) de 22 mulheres submetidas à cirurgia de tension-free vaginal tape (TVT) para correção da incontinência urinária de esforço.

\begin{tabular}{|c|c|c|c|c|c|c|}
\hline \multirow{2}{*}{ DVJUV } & \multicolumn{2}{|c|}{ Repouso } & \multicolumn{2}{|c|}{ Esforço } & \multicolumn{2}{|c|}{ Deslocamento } \\
\hline & Pré-operatório & Pós-operatório & Pré-operatório & Pós-operatório & Pré-operatório & Pós-operatório \\
\hline Média (mm) & 11,4 & 11,0 & 16,4 & 15,4 & 7,2 & 5,6 \\
\hline Desvio-padrão & 6,3 & 5,5 & 7,6 & 8,9 & 5,2 & 4,0 \\
\hline Significância & \multicolumn{2}{|c|}{$p=0,33$} & \multicolumn{2}{|c|}{$p=0,28$} & \multicolumn{2}{|c|}{$p=0,18$} \\
\hline
\end{tabular}

Tabela 3

Estatísticos relativas à distância pubouretral (DPU) de 22 mulheres submetidas à ciru rgia de tension-free vaginal tape (TVT) para correção da incontinência urinária de esforço.

\begin{tabular}{|c|c|c|c|c|c|c|}
\hline \multirow{2}{*}{ DVJUV } & \multicolumn{2}{|c|}{ Repouso } & \multicolumn{2}{|c|}{ Esforço } & \multicolumn{2}{|c|}{ Deslocamento } \\
\hline & Pré-operatório & Pós-operatório & Pré-operatório & Pós-operatório & Pré-operatório & Pós-operatório \\
\hline Média (mm) & 13,4 & 10,9 & 18,3 & 14,4 & 5,7 & 4,8 \\
\hline Desvio-padrão & 5 & 2,8 & 7,5 & 6,9 & 5,0 & 4,3 \\
\hline Significância & \multicolumn{2}{|c|}{$p=0,24$} & \multicolumn{2}{|c|}{$p=0,26$} & \multicolumn{2}{|c|}{$p=0,43$} \\
\hline
\end{tabular}

\section{Tabela 4}

Estatísticos relativas à uretra proximal (UP) de 22 mulheres submetidas à ciru rgia de tension-free vaginal tape (TVT) para correção da incontinência urinária de esforço.

\begin{tabular}{|c|c|c|c|c|c|c|}
\hline \multirow{2}{*}{ DVJUV } & \multicolumn{2}{|c|}{ Repouso } & \multicolumn{2}{|c|}{ Esforço } & \multicolumn{2}{|c|}{ Deslocamento } \\
\hline & Pré-operatório & Pós-operatório & Pré-operatório & Pós-operatório & Pré-operatório & Pós-operatório \\
\hline Média (mm) & 15,3 & 17,3 & 3,7 & 8,4 & 13,1 & 9,2 \\
\hline Desvio-padrão & 4,7 & 3 & 8,4 & 8,2 & 6,7 & 6,6 \\
\hline Significância & \multicolumn{2}{|c|}{$p=0,30$} & \multicolumn{2}{|c|}{$p=0,05$} & \multicolumn{2}{|c|}{$p=0,02$} \\
\hline
\end{tabular}

\section{Discussão}

Há numerosas divergências entre os pesquisadores tanto nos métodos para diagnóstico como no tratamento da IUE. 1,2 Entretanto, estudos mais recentes têm levado a um consenso de que as cirurgias de sling pubovaginal e as uretrocistopexias retropúbicas são as técnicas de tratamento da IUE que apresentam os melhores resultados com acompanhamento de longo prazo, quando comparadas com o tratamento convencional. ${ }^{3}$ Os slings pubovaginais, apesar de serem uma técnica relativamente simples, implicam 
na inconveniência da necessidade de um sitio de doação para a fáscia a ser utilizada, no caso de enxertos autólogos. E no caso do TVT, o qual conceitualmente corresponde a um sling, na utilização de um material sintético e por vezes dispendioso, além do risco de provocar obstrução infravesical e outras complicações. ${ }^{3-6}$

Teoricamente o TVT proporciona um aumento na resistência uretral por criar um suporte na região suburetral, evitando a descida rotacional da uretra (hipermobilidade), facilitando a coaptação de suas paredes. ${ }^{3-6}$

A avaliação, feita após 30 dias da realização da cirurgia, permitiu identificar que a cirurgia pela técnica de TVT efetivamente provocou melhora do quadro de IUE em $91 \%$ das pacientes que ficaram sem queixas no pós-operatório (IUE grau 0). Após essa primeira avaliação pós-operatória, duas pacientes (9\%) permaneceram com IUE leve (grau I), sendo que apenas uma delas permanecia com perda aos esforços após a última visita, decorridos três meses de cirurgia, o qual foi considerado como único insucesso do tratamento até aquele período.

Mesmo que se considere a parcialidade dessa avaliação, decorrente da subjetividade e da margem de variação devida à diferença de percepção de cada paciente, esse possível viés foi minimizado ao se considerar cada paciente como seu padrão.

Nesse estudo, dois dos parâmetros ultra-sonográficos selecionados funcionaram bem tanto na confirmação do diagnóstico no pré-operatório, como na avaliação pós-operatória, em conformidade com os resultados clínicos. Segundo dados apresentados no presente estudo, não há diferença estatisticamente significante entre as medidas ultra-sonográficas de deslocamento ao esforço no pré e pós-operatório, dos parâmetros DHJUV e DPU. Por outro lado os parâmetros UP $(p=0,02)$ e DVJUV $(p=0,005)$ mostraram-se com diferenças estatisticamente significantes no pós-operatório. O menor deslocamento, conseqüentemente com maior alongamento da uretra proximal (UP) no esforço, após a cirurgia, parece ser importante uma vez que a uretra funcional mais curta pode causar perda por não oferecer resistência, em extensão adequada, ao escape de urina. O deslocamento vertical da JUV (DVJUV), parâmetro mais aceito como indicador de hipermobilidade uretral, foi menor após a cirurgia de TVT nessa amostra, sendo essa diferença extremamente significante $(p=0,005)$. Uma medida maior ou igual a $10 \mathrm{~mm}$ de deslocamento da JUV tem sido comu- mente aceita como característica de hipermobilidade em pacientes com IUE.7-11

Nesse estudo, utilizando a ultra-sonografia transvulvar, a média de deslocamento vertical da JUV no pré-operatório foi de $16,7 \mathrm{~mm}$, o que confirma sua utilidade como método diagnóstico de hipermobilidade. Dessa forma, também, pode-se inferir que enquanto pelos estudos urodinâmicos afirma-se que o TVT aumenta a resistência uretral, pela avaliação ultra-sonográfica pode-se dizer que a referida cirurgia diminui a mobilidade da JUV e da UP e, efetivamente, alonga a uretra proximal no esforço. Persiste ainda uma questão para reflexão: no esforço, que provoca a perda urinária nas mulheres com IUE, o TVT atua aumentando o comprimento ou aumentando a resistência da UP?

Por outro lado, mesmo com a melhora subjetiva das queixas de IUE em todas as 22 pacientes tratadas com a cirurgia de TVT e ausência da queixa de perda urinária efetiva em mais de $90 \%$ das pacientes, a média de deslocamento vertical da JUV medidos quatro semanas após a cirurgia, diminuiu significantemente para 10,6 mm nesse grupo, tendo, porém permanecido acima dos $10 \mathrm{~mm}$, comumente aceitos como diagnóstico de hipermobilidade. Uma possível explicação para isso pode dever-se ao fato de que o TVT seria responsável na reposição ou reforço de estruturas ligamentares, restaurando uma anatomia mais funcional e provocando um "acotovelamento funcional" na altura da uretra média, e não tendo sua ação ao nível do colo vesical ou JUV, como outros procedimentos deslings ou reparos anteriores. 3-6

Mesmo com os resultados satisfatórios, acima de $90 \%$ e em conformidade com a literatura mundial,3-7 não se pode afirmar que as pacientes operadas pela cirurgia de TVT tenham sido definitivamente curadas, sendo necessário seguimento clínico muito mais prolongado. Entretanto, deve ficar claro que o intuito deste trabalho não é mostrar a eficácia do TVT, porque é necessário um tempo de aproximadamente 10 anos para se dizer com segurança que um procedimento cirúrgico para tratamento da IUE é eficaz ou sem recidiva.

\section{Conclusões}

A cirurgia do tipo TVT usada no tratamento de mulheres com incontinência urinária de esforço reduz a mobilidade vertical da junção uretrovesical e da uretra proximal. 


\section{Referências}

1. Hannestad YS, Rortveit G, Hunskaar S. Help-seeking and associated factors in female urinary incontinence. The Norwegian EPINCONT Study. Epidemiology of Incontinence in the County of Nord-Trondelag. Scand $\mathrm{J}$ Prim Health Care 2002; 20:102-7.

2. Minassian VA, Drutz HP, Al-Badr A. Urinary incontinence as a worldwide problem. Int J Gynaecol Obst 2003; 82: 327-38.

3. Brophy MM, Klutke JJ, Klutke CG. A review of the tension-free vaginal tape procedure: outcomes, complications, and theories. Curr Urol Rep 2001; 2: 364-9.

4. Carlin BI, Klutke JJ, Klutke CG. The tension-free vaginal tape procedure for the treatment of stress incontinence in the female patient. Urology 2000; 56: 28-31.

5. Ulmsten U, Falconer C, Johnson P, Jomaa M, Lanner L, Nilsson CG. A multicenter study of tension-free vaginal tape (TVT) for surgical treatment of stress urinary incontinence. Int Urogynecol J Pelvic Floor Dysfunct 1998; 9 : 210-3.

6. Ulmsten U, Johnson P, Rezapour M. A three-year follow up of tension free vaginal tape for surgical treatment of female stress urinary incontinence. Br J Obstet Gynaecol 1999; 106: $345-50$.
7. Brandt FT, Albuquerque CD, Lorenzato FR, Amaral FJ. Perineal assessment of urethrovesical junction mobility in young continent females. Int Urogynecol J Pelvic Floor Dysfunct 2000; 11: 18-22.

8. Bergman A, Vermesh M, Ballard CA, Platt LD. Role of ultrasound in urinary incontinence evaluation. Urology 1989; 33: 443- 4 .

9. Chen GD, Su TH, Lin LY. Applicability of perineal sonography in anatomical evaluation of bladder neck in women with and without genuine stress incontinence. J Clin Ultrasound 1997; 25: 189-94.

10. Granados Loarca EA, Alcahe VR, de Leon Lopez H, Echeverria Reyes J. The usefulness of perineal ultrasound in urinary incontinence in women. Arch Esp Urol 1999; 52: $778-8$

11. Virtanen HS, Kiilholma P. Urogynecologic ultrasound is a useful aid in the assessment of female stress urinary incontinence-a prospective study with TVT procedure. Int Urogynecol J Pelvic Floor Dysfunct 2002; 13: 218- 22.

Recebido em 27 de agos to de 2004

Versão final aprovada em 3 de março de 2005

Aprovado em 28 de abril de 2005 